\title{
Combination of Docetaxel with Thymoquinone in Nanoemulsion Impedes the Migration of Breast Cancer Stem Cells
}

\author{
Bawadud RS, Mayson H. Alkhatib*, Gashlan HM \\ Department of Biochemistry, Faculty of Science, King Abdulaziz University, Jeddah, SAUDI ARABIA.
}

\begin{abstract}
Objectives: Metastatic breast cancer is an advanced stage that is highly dependent on migration signaling pathways, though it remains unclear what mechanisms control the maintenance of tumorigenic cells that drives the migration and invasion processes. This in vitro study examined the intercellular cross-talk in MCF-7 and MDA-MB-231 breast cancer cells when treated with the simultaneous administration of docetaxel (DOC) and thymoquinone (TQ) loaded in nanoemulsion (NE) delivery system. Method: The cell cycle and breast cancer stem cells (BCSC) population were evaluated using flow cytometric analysis, alongside a DNA fragmentation assessment. Further molecular investigations were performed utilizing quantitative polymerase chain reaction (Q-PCR) technique. Results: The DOC+TQ/NE formulation inhibits the proliferation of human cancer cells by the $\mathrm{G}_{2} / \mathrm{M}$ phase and S-phase cell cycle arrest in MCF-7 and MDA-MB-231 cells, receptively, and it stimulates apoptosis through induction of DNA damage. Moreover, the DOC+TQ/NE causes a significant reduction in the cellular migration activity in both cell lines as detected by the wound-healing assay. This observation could possibly be mediated through the inhibition of BCSC, accompanied by marked down-regulation
\end{abstract}

of SNAIL-1 and TWIST-1 expression. Conclusion: Overall, the results demonstrate that the co-delivery of DOC and TQ within NE formulation might be considered as a promising and effective therapeutic approach for breast cancer that could prevent the intercellular signaling function of metastasis.

Key words: Apoptosis, EMT signaling pathways, Cell cycle arrest, DNA fragmentation.

Correspondence

Mayson H. Alkhatib,

Department of Biochemistry, Faculty of Science, King Abdulaziz University, Jeddah, SAUDI ARABIA.

Phone: +966599240526

Email: mhalkhatib@kau.edu.sa;

ORCID: http://orcid.org/0000-0002-3729-5303

DOI: 10.5530/ijpi.2020.2.39

\section{INTRODUCTION}

The metastatic process is the leading characteristic of aggressive malignant breast cancer cells. ${ }^{1}$ Despite various developed treatments, the tumor recurrence in breast cancer patients remains a major complication of conventional therapy. Failure in addressing the root causes of the metastasis process would eventually lead to breast cancer relapse. During cancer progression, a cell morphologic conversion process occurs which is known as an epithelial-mesenchymal transition (EMT), a cellular process that transforms polarized epithelial cells to non-polarized and invasive mesenchymal cells. ${ }^{2}$ Recent evidence suggests that a small population of stem-like cancer cells possess specifically extensive selfrenewal and migratory potentials that contribute to the metastatic growth in breast cancer.

The EMT signaling pathways are closely entangled with the development and maintenance of breast cancer stem cells (BCSC) that display cellular plasticity. $^{3}$ As most standard therapeutic agents are proposed mainly to interfere with rapidly dividing cells, these tumorigenic stem cells would be highly resistant to chemotherapy. ${ }^{4}$ Therefore, it is necessary to find effective new therapeutic approaches that have the ability to control local metastasis by targeting the BCSC and overcome the major limitations of traditional therapy.

Greener synthesis and fabrication of nanomaterials recently have gained growing attention due to their exclusive characteristics towards biomedical applications as an alternative to overcome the toxic effects of conventional therapy. ${ }^{5}$ Oil-in-water $(\mathrm{o} / \mathrm{w})$ nanoemulsions, where the oil phase is dispersed uniformly as nanosized droplets in the water phase, have been widely studied in the fields of encapsulation and targeting to enhance the poorly soluble drugs bioavailability. The small droplet size allows better adherence to membranes, leading to improvements in the cellular uptake of bioactive molecules. ${ }^{6}$ Borage, the richest known plant source of the gamma-linolenic acid (GLA), a type of omega- 6 fatty acid has gained a renewed interest because of its antioxidant and anti-inflammatory therapeutic properties that would enhance the cancer prevention. ${ }^{\text {? }}$

Combination therapy of bioactive natural products with anticancer drugsrepresentsapromisingapproach thatmayenhancechemotherapeutic potentiality. Thymoquinone (TQ) is the main bioactive component of Nigella sativa L. Seeds Essential Oil, which is mostly responsible for the unique therapeutic properties. ${ }^{8}$ Owing to its anticancer activity, it has been widely used as an adjuvant to chemotherapeutic agents in order to minimize its dose and accordingly associated risks of side effects in breast cancer.

Therefore, the aims of the present study were to examine the antitumor effect of borage-based NE co-loaded with Docetaxel (DOC) a taxane antimitotic chemotherapy and TQ in MCF-7 and MDA-MB-231 the human breast cancer cell lines. The research investigation focused on two central questions, firstly whether the mechanisms of the co-delivery of DOC and TQ within the NE effect involved the regulation of cell cycle and apoptosis. Secondly, whether or not it could restrain the migration through the inhibition of BCSC and interference with EMT signaling cascades. 


\section{MATERIALS AND METHODS}

\section{Materials}

Docetaxel was kindly supplied by King Abdulaziz University Hospital. Thymoquinone was obtained from Sigma-Aldrich (USA). The oils of borage and medium-chain triacylglycerols (MCT) were obtained from the electronic store iHerp.com. The surfactant Tween 80, cosurfactant Span 20 and pH8 buffer solution were obtained from Al-Shafei Medical and Scientific Equipment, Est (Jeddah, KSA). Dulbecco's modified eagle medium (DMEM), fetal bovine serum (FBS), trypsin and penicillinstreptomycin were purchased from Gibco (Grand Island, NY, USA). Propidium Iodide was obtained from Cayman's Chemical (Michigan, US). Human breast cancer cell lines, MCF-7 and MDA-MB-231, were procured from America Type Tissue Culture Collection (Manassas, VA, USA).

\section{Synthesis of Nanoemulsion (NE)}

Oil-in-water $(\mathrm{O} / \mathrm{W})$ nanoemulsion was formulated by adding a combination of the oil phase of $5.55 \%(\mathrm{v} / \mathrm{v})$ of borage and medium-chain triglyceride (MCT) oils into the aqueous phase of $75 \%(\mathrm{v} / \mathrm{v})$ of buffer solution ( $\mathrm{pH} 8)$. Then, the warm emulsifying agents of $19.45 \%(\mathrm{v} / \mathrm{v})$ of Tween 80 and Span 20 mixed at a constant ratio of (2:1), respectively, were added and the ratio between oils to surfactants $\mathrm{O} / \mathrm{S}$ was kept at (1: 3. 5) since it is the optimal stability of the produced formula. The mixture appeared as a single uniform phase with milky-white color that was then further homogenized with a sonicator (Omni Sonic Ruptor 4000 Ultrasonic Homogenizer, Kennesaw, GA, USA) until a clear and transparent solution gets formed demonstrating the production of nanoemulsion. For the formulation of DOC+TQ/NE samples, the stock solutions of $100 \mu \mathrm{M}$, prepared by mixing docetaxel and thymoquinone at a ratio of (1:1) and then directly added dropwise along with vortexing into a freshly prepared blank-NE. Following, the resulted combination was further diluted to the desired intermediate concentrations depending on each experiment.

\section{Cell Cultures}

The human breast cancer cell lines (MCF-7 and MDA-MB-231) were cultured in complete medium (DMEM), supplemented with $10 \%(\mathrm{v} / \mathrm{v})$ FBS and $1 \%(\mathrm{v} / \mathrm{v})$ penicillin-streptomycin, and maintained in a humidified cell culture incubator at $37^{\circ} \mathrm{C}$ and in $5 \% \mathrm{CO}_{2}$. To sustain the optimum growth and viability of cultured cells when reached $90 \%$, confluent cells were frequently sub-cultured by trypsinization. Both cell lines were diluted to the desired seeding density depending on each experiment.

\section{Cell cycle distribution analysis}

Cells were seeded into a 6-well plate until 70\% confluence and after $48 \mathrm{~h}$ of treatment, collected cells were fixed with ice-cold $70 \%$ ethanol at $-20^{\circ} \mathrm{C}$ overnight. Then, washed cells pellets were re-suspended in $1 \mathrm{~mL}$ of PBS including $50 \mathrm{~g} / \mathrm{mL}$ RNase A with 10 propidium iodide (PI). All samples were then incubated for $20 \mathrm{~min}$ in the dark at $37^{\circ} \mathrm{C}$. The cell cycle profiles analysis was performed using the flow cytometry (BD FACSAria $^{\text {ma }}$ III, San Jose, CA 95131, United States). The collected data were analyzed by FACS Diva Version 6.1.3 software.

\section{Quantification of DNA fragmentation}

The DNA fragmentation was assessed with an ELISA "enzymelinked immunosorbent assay" Cell Death Detection kit (Roche Molecular Biochemicals, Mannheim, Germany), according to the manufacturer's instructions. The DNA fragmentation was measured spectrophotometrically at $405 \mathrm{~nm}$ (reference $\lambda=490 \mathrm{~nm}$ ) and the ratio of fragmentation versus controls was then calculated. The enrichment factor was assessed as a parameter of apoptosis and plotted on the y-axis as the mean $\pm \mathrm{SD}$ of three independent experiments.

\section{Cell culture wound healing assay}

Cells were seeded in a 12 -well plate and allowed to reach $100 \%$ confluence. The following day, a vertical wound down through the cell monolayer was created by using a $200 \mu \mathrm{L}$ pipette tip in a sterile environment. After treatments, an initial picture was taken for each sample at 10x magnification using computer-assisted intravital microscopy (TH4-200, Olympus optical Co-Ltd, Japan). Then, all generated wounds were allowed to recover in the incubator for further $24 \mathrm{~h}$ and $48 \mathrm{~h}$. The wound closure was assessed by imaging and measuring the width of the wound over time using the Image J program.

\section{Breast cancer stem cells detection}

The effects on BCSC (CD44 $\left.4^{\text {high }} / \mathrm{CD} 24^{\text {low }}\right)$ were assessed using flow cytometry coupled with FITC labeled mouse monoclonal [B-F24] anti-CD44 and APC/Cy7 labeled mouse monoclonal [SN3] anti-CD24 antibodies (Abcam Inc., Cambridge Science Park, Cambridge, UK). In general, treated cells for $48 \mathrm{~h}$ were collected and washed with $10 \%$ FBS in ice-cold PBS. Afterward, cells were resuspended in the $5 \mu \mathrm{L}$ of $0.1 \mu \mathrm{g} / \mathrm{mL}$ conjugated anti-CD44 and $5 \mu \mathrm{L}$ of $0.1 \mu \mathrm{L}$ anti-CD24 antibodies and incubated in the dark for $30 \mathrm{~min}$. The control cells were incubated with $5 \mu \mathrm{L}$ of mouse IgG1, monoclonal [HyblgG1] (FITC) - isotype control, and $5 \mu \mathrm{L}$ of mouse IgG1, kappa monoclonal (APC/CY7) - isotype control (Abcam, Cambridge, UK). After the staining step, cells were washed three times with 10\% FCS in ice-cold PBS and were then analyzed by Flow Cytometer (BD FACSAria ${ }^{\mathrm{Tm}}$ III, San Jose, CA 95131, United States) using FACS Diva Version 6.1.3 software.

\section{Quantitative PCR analysis}

A reverse transcription-quantitative polymerase chain reaction (RT-qPCR) was performed to evaluate the expression levels of SNAIL-1 and TWIST-1 genes in MCF-7 and MDA-MB-231 cells after treatment for $48 \mathrm{~h}$. Briefly, total RNA of treated cells was extracted using the Qiagen RNeasy Mini Kit (Qiagen, Hilden, Germany) and quantified spectrophotometrically. Subsequently, single-strand cDNA was synthesizedaccordingtothemanufacturer'sinstructionsofaHigh-Capacity cDNA Reverse Transcription Kit (Applied Biosystems, CA, USA). Sequences of all primers used are listed below in Table 1 . The selected gene expression was then quantified by using the SYBR Green PCR master mix (Applied Biosystems) on a Step One Plus ${ }^{\text {mix }}$ Real-Time PCR System (Applied Biosystems, USA). Upon analysis, acquired results were normalized to the endogenous reference GAPDH and calculated by the $2^{-\Delta \Delta \mathrm{Ct}}$ method to determine the relative mRNA expression of target genes.

\section{Statistical analysis}

All experiments were performed at least in triplicate and measurements were reported as the mean \pm standard deviation (SD). Data were analyzed by one-factor analysis of variance (ANOVA) using Prism, version 8.00, for Mac OS X (GraphPad Software, CA, USA). Results were considered statistically significant at $p<0.05$.

\section{RESULTS}

\section{Cell cycle distribution}

The percentage of DNA at $G_{1}, S$, and $G_{2} / M$ phases of the cell cycle was evaluated using PI staining and flow cytometry. According to Figure 1, there were no significant changes in the cell cycle of the MCF-7 cells treated with TQ. However, a significant arrest at the $\mathrm{G}_{2} / \mathrm{M}$ phase following treatment with both free-DOC and the loaded DOC+TQ/NE was determined. The S-phase arrested cell significantly increased when exposed to free-NE. In contrast, the effect of DOC-free and the loaded DOC+TQ/NE was significantly profound on the S-phase of the cell cycle distribution of MDA-MB-231 cells. In addition, the number of cells arrested at S-phase was the highest following treatment with free-NE. 
Table 1: Sequences of primers used in the study.

\begin{tabular}{cccc}
\hline Gene & Forward primer $\left(\mathbf{3}^{\prime}-5^{\prime}\right)$ & Reverse primer $\left(5^{\prime}-\mathbf{3}^{\prime}\right)$ & Product length $(\mathbf{b p})$ \\
\hline GAPDH & GACAGTCAGCCGCATCTTCT & GCGCCCAATACGACCAAATC & $80-120$ \\
TWIST-1 & CGTGGGGCGCACTTTTAAAA & CAGAGGTGTGAGGATGGTGC & $80-120$ \\
SNAIL -1 & GAATTCCCTCCTGAGTGCCC & CAGGCAGAGGACACAGAACC & $80-120$ \\
\hline
\end{tabular}

(A)

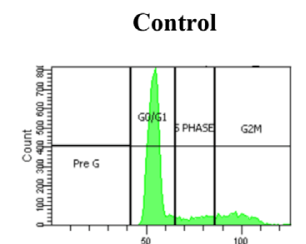

Free-NE

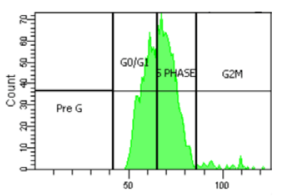

(B)

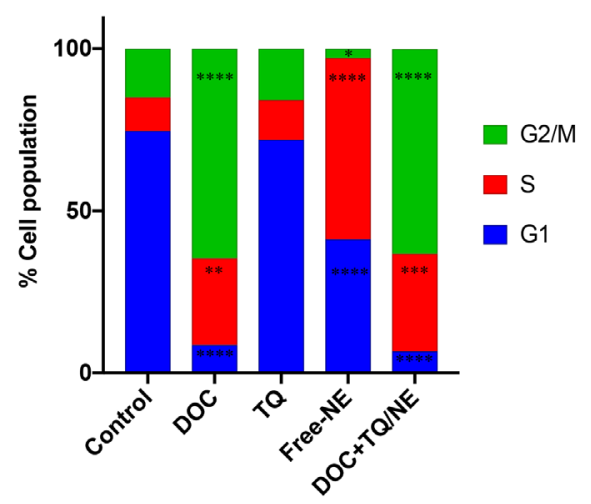

Figure 1: Effect on cell cycle distribution of MCF-7 cells after 48h. (A) cell cycle distribution was determined using flow cytometry analysis post PI staining. (B) Different cell phases (G1, S, and G2/M) were plotted as percentage of total events. Data is presented as mean $\pm S D, n=3, * P 0.033,{ }^{* *}$ $P<0.0021$, ${ }^{* * *} P<0.0002,{ }^{* * * *} P<0.0001$ in comparison to control.

Representative percentages of MDA-MB-231 cells in $G_{1}, S$ and $G_{2} / M$ phase are displayed in Figure 2.

\section{DNA fragmentation assessment}

A quantitative analysis of the cytoplasmic histone-associated DNA fragmentation using the ELISA sandwich-enzyme-immunoassay was performed. As illustrated in Figure 3 (A and B), the findings indicated a marked increase in the level of DNA fragmentation when both cell lines were treated with either free-DOC or loaded DOC+TQ/NE. However, the effect on cells seemingly was less pronounced with free-TQ and NE treatments, suggesting that cells undergoing apoptosis were lower than free-DOC.

\section{Inhibition of migration capacity of MCF-7 and MDA-MB-231 cells}

To verify whether the formulated treatment could decrease the metastatic potential of the tested breast cancer cell lines, wound healing assay was performed as shown in Figure 4 (A) and Figure 5 (A). In MCF-7 cells, the
(A)

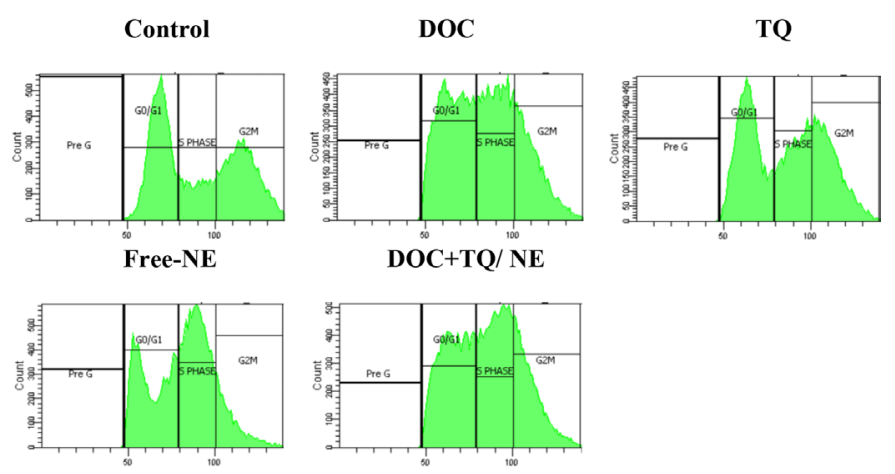

(B)

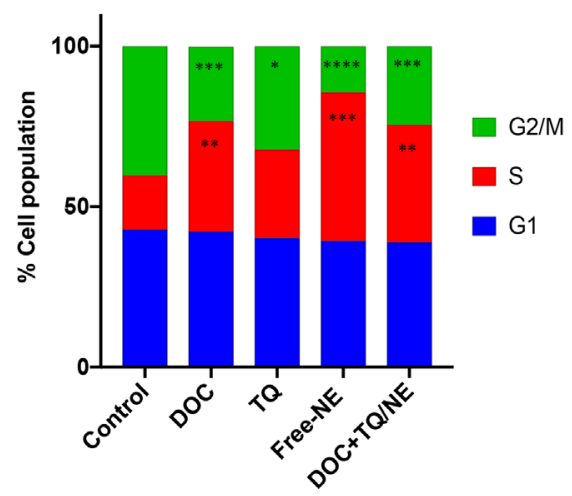

Figure 2: Effect on cell cycle distribution of MDA-MB-231 cells after 48h. (A) cell cycle distribution was determined using flow cytometry analysis post PI staining. (B) Different cell phases (G1, S, and G2/M) were plotted as percentage of total events. Data is presented as mean $\pm \mathrm{SD}, \mathrm{n}=3,{ }^{*} P 0.033^{* *}$ $P<0.0021,{ }^{* * *} P<0.0002,{ }^{* * * *} P<0.0001$ in comparison to control.

achieved data demonstrated that TQ and free-NE reduced the migration ability of cells. However, the combination of DOC+TQ/NE demonstrated a significantly greater effect on the inhibition of migration rates than any of the treatments alone (Figure 4B). In addition, the half-closure time analysis, which showed the point at which the wound is half the original area, further confirmed the significant effect of DOC+TQ/NE as illustrated in Figure 4C. Furthermore, the findings displayed a significant decrease in the migration of MDA-MB-231 cells when treated with the loaded DOC+TQ/NE compared to untreated control cells. On the other hand, the single treatments did not exhibit an inhibitory effect on the migration rate of MDA-MB-231 cells as shown in Figure 5B. Moreover, the half-closure time of MDA-MB-231cells treated with either free DOC or $\mathrm{DOC}+\mathrm{TQ} / \mathrm{NE}$ was found to be higher than other treatments compared to control cells (Figure 5C). 
(A)

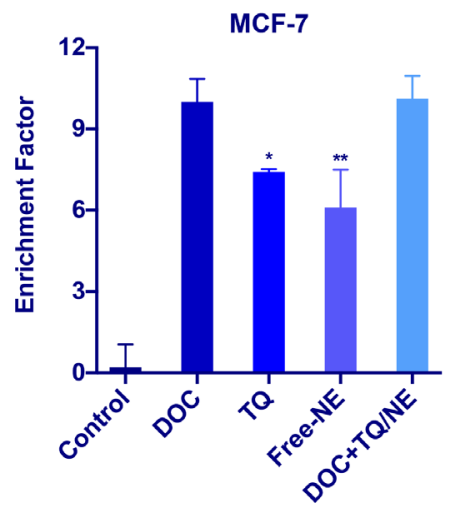

(B)

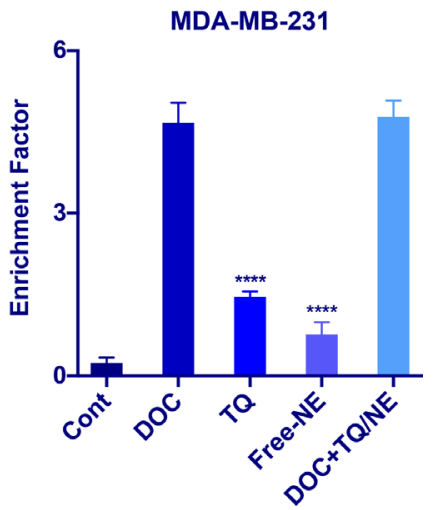

Figure 3: Effect on the enrichment of nucleosomes in the cytoplasm after 48h. Enrichment factor was determined by ELISA assay as indicated in the manufacture protocol in (A) MCF-7 and (B) MDA-MB-231 cells. At the end of the assay, the absorbance at $(405-490 \mathrm{~nm})$ was red on a microplate reader. Data is presented as mean $\pm S D ; n=2$. Significant differences of all treatments were analyzed by one-way ANOVA *P<0.033, ${ }^{* *} \mathrm{P}<0.0021,{ }^{* * *} P<0.0001$ Versus DOC.

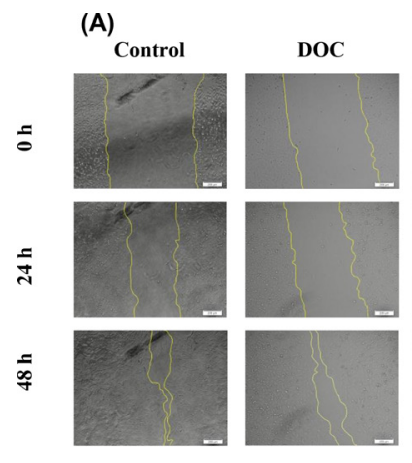

(B)

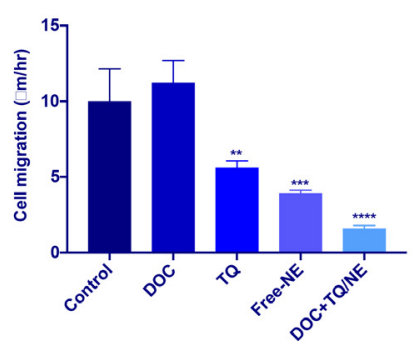

(C)

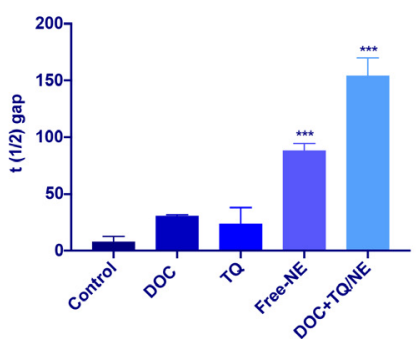

Figure 4: The wound healing assay can reveal differences in migration rates in MCF-7 cells. (A) Images from wound healing assay using phase contrast microscopy at different time points (0h, 24h and 48h). Scale bar $=200 \mu \mathrm{m}$. (B) Quantification of the average migration rate in MCF-7 cells from the acquired images by using the image J program. (C) Shows the calculated values, the time at which the gap is half the original area. Data is presented as mean \pm SD; $n=3$. ${ }^{* *} P<0.0021,{ }^{* * *} P<0.0002,{ }^{* * * *} P<0.0001$ in comparison to control.

\section{BCSC subpopulation reduction}

To investigate whether the suggested combination could eliminate the MCF-7 and MDA-MB-231 cells with the stem cell characteristics, flow cytometric analysis was performed to detect BCSC characterized by the CD $44^{\text {high }} / \mathrm{CD} 24^{\text {low }}$ expression pattern. It was found that in both cell lines, TQ alone did not alter the percentage of CD $44^{\text {high }} / \mathrm{CD} 24^{\text {low }}$ cell populations. However, MCF-7 treated cells with free-DOC and DOC+TQ/NE exhibited a significant decrease in the $\mathrm{CD} 44^{\text {high }} / \mathrm{CD} 24^{\text {low }}$ population
(A)

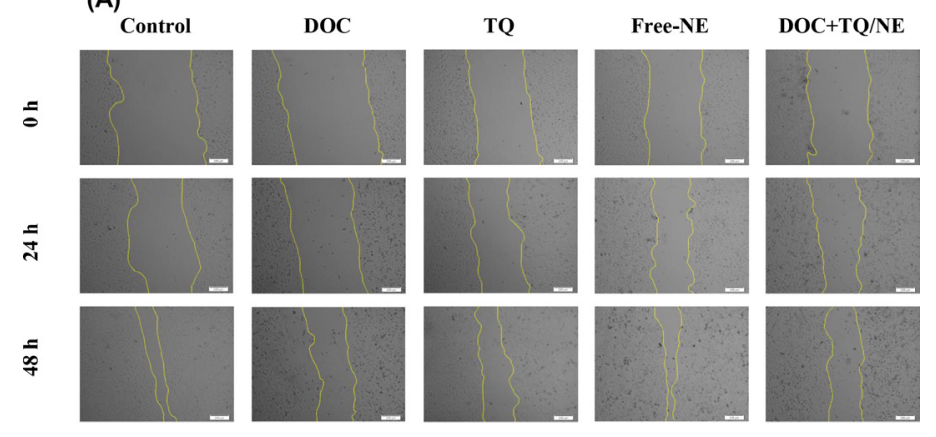

(B)

(C)
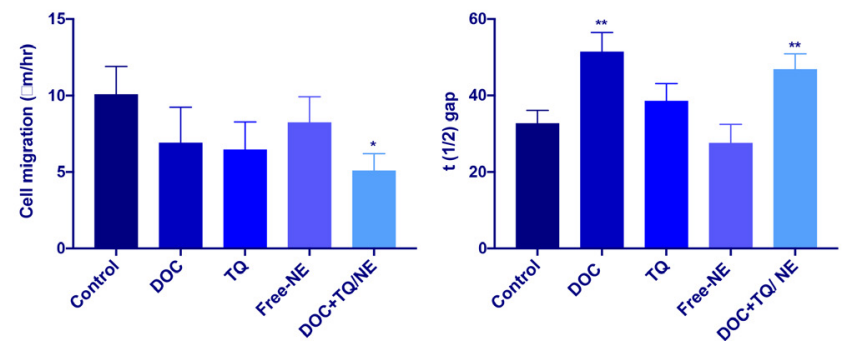

Figure 5: The wound healing assay can reveal differences in migration rates in MDA-MB-231cells. (A) Images from wound healing assay using phase contrast microscopy at different time points ( $0 \mathrm{~h}, 24 \mathrm{~h}$ and $48 \mathrm{~h}$ ). Scale bar $=200 \mu \mathrm{m}$. (B) Quantification of the average migration rate in MDA-MB-231 cells from the acquired images by using the image J program. (C) Shows the calculated values, the time at which the gap is half the original area. Data is presented as mean $\pm \mathrm{SD} ; n=3 .{ }^{* *} P<0.0021,{ }^{*} P 0.033$ in comparison to control.
(A)

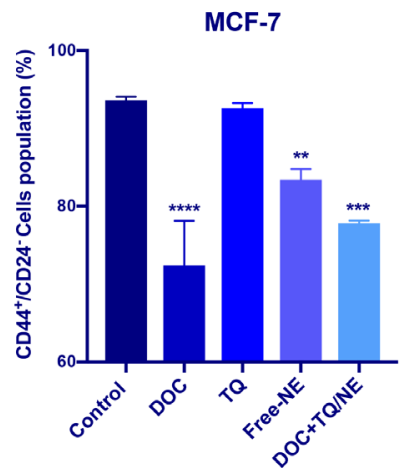

(B)

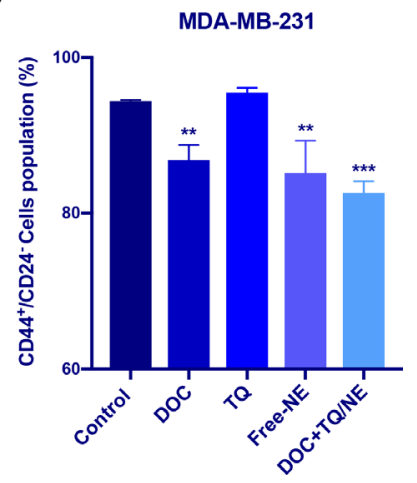

Figure 6: The effects on cancer stem cell clone after $48 \mathrm{~h}$. Cells were stained with anti-CD44, and anti-CD24 antibodies. (A) and (B) a summary of the percentage of $\mathrm{CD}_{4} 4^{+} / \mathrm{CD} 24$ cell clone after treatment is shown in MCF-7 and MDA-MB-231 cells, respectively. Data is presented as mean $\pm \mathrm{SD} ; n=3$. ** $P<0.0021$, ${ }^{* * *} P<0.0002,{ }^{* * * *} P<0.0001$ in comparison to control.

compared with untreated control cells. Furthermore, in MDA-MB231cells, DOC+TQ/NE treatment significantly potentiated the depletion of $\mathrm{CD} 44^{\text {high }} / \mathrm{CD} 24^{\text {low }}$ populations by $12.5 \pm 0.08 \%$ compared to untreated cells which were considerably greater than the decrease caused by freeDOC $(8 \pm 0.1 \%)$. All representative results are shown in Figure 6 (A and B).

\section{Assessment of EMT genes expression}

According to the RT-qPCR assessment in MCF-7 cells illustrated in Figure $7 \mathrm{~A}$, it is noticed that the expressions of TWIST-1 and SNAIL-1 
(A)

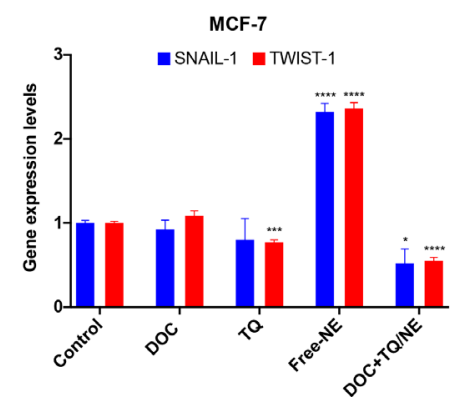

(B)

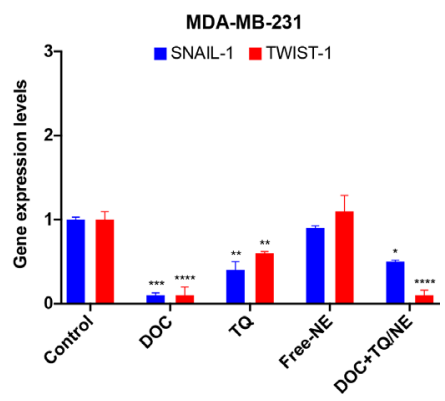

Figure 7: Effect on the expression of EMT related genes in cells after 48h. In (A) MCF-7 and (B) MDA-231 cells, the total RNA is shown that was extracted and subjected to RT-qPCR to measure gene expression. Data was plotted using $2^{-\Delta \Delta c t}$ method (expression normalized to the house keeping gene GAPDH). Data presented as mean $\pm \mathrm{SD} ; \mathrm{n}=3$. * $P$ 0.033, ** $P<0.0021$, *** $P<0.0002,{ }^{* * * *} P<0.0001$ compared to untreated control cells.

were not altered after exposure to free-DOC treatment. However, exposure to TQ significantly decreased TWIST-1 expression by $0.77 \pm 0.03$ fold, while free-NE increased unexpectedly the expression of both SNAIL- 1 and TWIST-1 by $2.32 \pm 0.10$ fold and $2.36 \pm 0.07$ fold, respectively, compared to untreated control. Moreover, the expression of TWIST-1 was significantly decreased after exposure to the combination of DOC+TQ/NE by $0.55 \pm 0.04$ fold compared to untreated control.

Ontheotherhand,inMDA-MB-231 cells, thefree-NEshowed nosignificant changes in the expression of TWIST-1 and SNAIL-1 as determined by RT-qPCR, while free-DOC treatment significantly decreased both TWIST-1 and SNAIL- 1 expressions by $0.05 \pm 0.03$ fold and $0.05 \pm 0.10$ fold, respectively. Additionally, the TQ treatment exhibited a significant decrease in the expressions of SNAIL- 1 and TWIST- 1 by $0.44 \pm 0.37$ fold and $0.57 \pm 0.02$ fold, respectively. Noteworthy, similarly to MCF-7 cells, when MDA-MB-231 cells treated with DOC+TQ/NE formulation, the expression of TWIST- 1 was inhibited markedly by $0.10 \pm 0.06$ fold compared to untreated control cells (Figure 7B).

\section{DISCUSSION}

In drug delivery, it could be a challenge to encapsulate different compounds into nanoemulsion system, since they might not be compatible which would result in more complications. However, several studies showed the effectiveness of DOC and TQ combination against cancer. ${ }^{9,10}$ Therefore, this study investigates whether the encapsulation of DOC and TQ into nanoemulsion would result in improved efficacy in terms of the inhibition of proliferation and migration of MCF-7 and MDA-MB-231 cells.

The type of cell death is an essential tool to determine the drugs mechanism of action. The DOC+TQ/NE formulation caused a significant $G_{2} / M$ phase arrest in MCF-7 cells, indicating that it would push cells to proliferate faster without DNA integrity checking or repair, allowing thus a damaged cell to enter mitosis and undergo apoptosis. ${ }^{11}$ In contrast, the DOC+TQ/NE induced a significant increase in the percentage of MDA-MB-231 cells arrested in S-phase of the cell cycle, suggesting its potential of interfering with the DNA synthesis phase that might result in cell death. Similar findings were previously reported that MDA-MB-231 cell proliferation was inhibited through the induction of $S$ phase arrest ${ }^{12}$. Moreover, the differences in the arrested phase of the cell cycle between MCF-7 and MDA-MB-231cells most probably attributed to differences in the treatment mechanism of actions that could lead tumor cells to be more susceptible to apoptosis. ${ }^{13}$
The DNA fragmentation evaluation revealed that DOC+TQ/NE combination caused significant DNA damage in both cell lines, suggesting the contribution of apoptosis induction to its mechanism of action. Additionally, the observed high level of DNA damage could clarify the activation of cell cycle arrest at various points in MCF-7 and MDAMB-231. Although, the concentration of DOC was lowered by third in the DOC+TQ/NE formula compared to free-DOC, interestingly it did not reduce its performance in inhibiting tumor cell proliferation with a high level of DNA fragmentation. This finding could indicate that as a strategy, the loaded drugs combination into the nanoemulsion system has the potential to reduce the drug dosing without compromising its clinical efficacy. ${ }^{14}$

Additionally, it is worth mentioning that the encapsulation of DOC+ TQ/NE was more powerful in limiting cancer cell growth than free-TQ as indicated by the high levels of DNA fragmentation. One explanation could be related to the major drawback of TQ, its high hydrophobicity resulting in poor bioavailability, thus, it cannot be used as the primary therapeutic agent. ${ }^{15}$ Therefore, the encapsulation within the nano delivery system would improve TQ efficiency.

The increased expression of the EMT-related transcription factors that strongly linked to BCSC play a critical role in the migration capabilities of cancer cells. ${ }^{3,16}$ According to the wound healing analysis, the migration ability of MCF-7 cells was markedly inhibited by the loaded combination of DOC+TQ/NE, and it was found to be more effective than free-DOC. Similarly, the migration rate of MDA-MB-231 cells was less affected by the single treatments. However, it exhibited a significant decrease when treated with $\mathrm{DOC}+\mathrm{TQ} / \mathrm{NE}$ combination. In addition to the previously found antiproliferation effect, this observation of cell migration further confirms the potential role of the co-loaded DOC and TQ within nanoemulsion formula as an effective approach for breast cancer therapy. Although some previous studies have confirmed the antitumor effect of DOC and TQ combination on breast cancer, to our knowledge this is the first study that demonstrates the effect of this combination when it encapsulated in the NE system on BCSC. The previous migration inhibition results could be attributed to the effect of DOC+TQ/NE formula to potentiate the depletion of BCSC clone, as indicated from the analysis of cell populations with the $\mathrm{CD} 44^{\text {high }} / \mathrm{CD} 24^{\text {low }}$ expression pattern in MCF-7 and MDA-MB-231 cells. This finding is in agreement with previous study that reported the enhanced selectivity toward BCSC of nanomedicine, which could explain the inhibition capacity of these treatments against cell migration. ${ }^{17}$

At the molecular level, the effect on the expression profile of EMT genes showed that SNAIL-1 levels were depleted after DOC+TQ/NE treatment in MDA-MB-231 and MCF-7 cells. The expression of SNAIL pathway has an essential role in the induction of EMT and metastasis, thus identifying therapeutics strategies which capable of downregulating SNAIL would be of great interest. ${ }^{16}$ Moreover, TWIST-1 was inhibited markedly by DOC+TQ/NE in both cell lines, suggesting that its downregulation may be contributed to the inhibited effect of tumor cell migration. ${ }^{3}$ A previous report suggested that TWIST depletion might be a promising approach against cancer progression and chemoresistance. ${ }^{18}$ It could be concluded that the effect of DOC+TQ/NE formulation on the migration capability of MDA-MB-231 and MCF-7 cells may probably be mediated by the reduction of SNAIL-1 and TWIST-1 expressions alongside the inhibition of BCSC population, as these factors play apparently distinct roles in metastasis and cancer progression.

\section{CONCLUSION}

The simultaneous administration of DOC and TQ co-loaded in nanoemulsion were proposed as an attempt to overcome the metastatic 
potential of breast cancer. This co-delivery system is mediated via several aspects that were regulated by diverse pathways. These aspects mainly include the regulation of breast cancer cell proliferation and metastasis. In this study, several pathways and mechanisms were highlighted that were probably governing the $\mathrm{DOC}+\mathrm{TQ} / \mathrm{NE}$ formulation antiproliferative and apoptotic effects such as the regulation of cell cycle through $\mathrm{S}$ phase arrest and $\mathrm{G}_{2} / \mathrm{M}$ phase arrest in MCF-7 and MDA-MB-231, respectively. The induction of the apoptotic pathway is owing to promoting DNA damage. In addition, the inhibition of migration was indicated by the reduction in the $\mathrm{BCSC}$ population and the diminishing in the expression of EMT-regulated gene products SNAIL-1 and TWIST-1. It was clear that all these aspects and pathways collaborate and interact together to mediate the anticancer effect of DOC+TQ/NE. Thus, using a nanoemulsion system for the co-delivery of DOC and TQ may offer a highly promising therapeutic approach that could prevent migration and force depletion of tumorigenic breast cancer stem-like cells.

\section{REFERENCES}

1. Lozano MC, González AG, Marchal JA, Muela EM, Molina MP, Cara FE, et al. Hydroxytyrosol inhibits cancer stem cells and the metastatic capacity of triplenegative breast cancer cell lines by the simultaneous targeting of epithelial-tomesenchymal transition. Wnt / $\beta$-catenin and TGF $\beta$ signaling pathways. Eur $J$ Nutr. 2018;1:13

2. Kalluri R, Weinberg RA. The basics of epithelial-mesenchymal transition. J Clin Invest. 2009;119(6):1420:8.

3. Kotiyal S, Bhattacharya S. Breast cancer stem cells, EMT and therapeutic targets. Biochem Biophys Res Commun. 2014;453(1):112:6.

4. Dey P, Rathod M, De A. Targeting stem cells in the realm of drug-resistant breast cancer. breast cancer targets and therapy. 2019;11:115:35.

5. Kharissova OV, Kharisov BI, Máximo C, González O, Méndez YP, López I. Greener synthesis of chemical compounds and materials. R Soc Open Sci. 2019;6(11):191378.
6. Fan Y, Zhang Y, Yokoyama W, Yi J. Endocytosis of Corn Oil-Caseinate Emulsions in vitro: Impacts of Droplet Sizes. Nanomaterials. 2017;7(11):349.

7. Lozano-Baena MD, Tasset I, Muñoz-Serrano A, Alonso-Moraga Á, Haro-Bailón A de. Cancer Prevention and Health Benefices of Traditionally Consumed Borago officinalis Plants. Nutrients. 2016;8(1):48.

8. Khan A, Tania M, Fu S, Fu J. Thymoquinone, as an anticancer molecule : from basic research to clinical investigation. Oncotarget. 2017;8(31):51907:19.

9. Sutton KM, Greenshields AL, Hoskin DW, Sutton KM, Greenshields AL, Hoskin DW. Thymoquinone, A Bioactive Component of Black Caraway Seeds, Causes G1 Phase Cell Cycle Arrest and Apoptosis in Triple-Negative Breast Cancer Cells with Mutant p53. Nutr Cancer. 2014;66(3):408:18.

10. Dirican A, Atmaca H, Bozkurt E, Erten C, Karaca B, Uslu R. Novel combination of docetaxel and thymoquinone induces synergistic cytotoxicity and apoptosis in DU-145 human prostate cancer cells by modulating PI3K - AKT pathway. Clin Transl Oncol. 2015;17(2):145:51.

11. Tyagi AK, Strongly S, Human S. To Arrest or Not To G2-M Cell-Cycle Arrest. Clin Cancer Res. 2002;8(11):3311:4.

12. Luo H, Wang F, Bai Y, Chen T, Zheng W. Colloids and Surfaces B : Biointerfaces Selenium nanoparticles inhibit the growth of HeLa and MDA-MB-231 cells through induction of $S$ phase arrest. Colloids Surf B Biointerfaces. 2012;94:304:8.

13. Schwartz GK, Shah MA. Targeting the Cell Cycle: A New Approach to Cancer Therapy. J Clin Oncol. 2005;23(36):9408:21.

14. Akhtartavan S, Karimi M, Karimian K, Azarpira N, Khatami M, Heli H. Evaluation of a self-nanoemulsifying docetaxel delivery system. Biomed Pharmacother. 2019;109:2427:33.

15. Elmowafy M, Samy A, Raslan MA, Salama A, Said RA, Abdelaziz AE, et al. Enhancement of Bioavailability and Pharmacodynamic Effects of Thymoquinone Via Nanostructured Lipid Carrier (NLC) Formulation. AAPS PharmSciTech. 2016;17(3):663-72.

16. Wu Y, Zhou BP. TNF- a / NF- k B / Snail pathway in cancer cell migration and invasion. Br J Cancer. 2010;102(4):639:44.

17. $\mathrm{NK} \mathrm{H}, \mathrm{DH} \mathrm{S}, \mathrm{JS} \mathrm{K}, \mathrm{KY}$ W, $\mathrm{CY}$ J, JS K. Hyaluronan-conjugated liposomes encapsulating gemcitabine for breast cancer stem cells. Int J Nanomedicine. 2016;11:1413:25.

18. Zhuo X, Chang A, Huang C, Yang L, Zhao H, WuY, et al. Nanoparticle-mediated down-regulation of TWIST increases radiosensitivity of nasopharyngeal carcinoma cells via ERK pathway. Am J Cancer Res. 2015;5(4):1571:9.

Article History: Submission Date : 24-02-2020; Revised Date : 03-03-2020; Acceptance Date : 23-03-2020

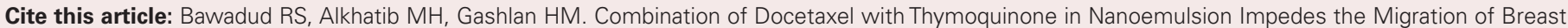
Cancer Stem Cells. Int. J. Pharm. Investigation. 2020;10(2):211-6. 